\title{
Eddington capture sphere around luminous relativistic stars
}

\author{
Maciek Wielgus \\ Nicolaus Copernicus Astronomical Center, PL-00-716, Warszawa, Poland \\ email: maciek.wielgus@gmail.com
}

\begin{abstract}
We discuss the interplay of gravity and radiation in a static, spherically symmetric spacetime. Because of the spacetime curvature, balance between radiation pressure from spherical star and effective force of gravity may be established in a particular distance from the star surface, on so-called Eddington capture sphere. This is in contrast with the Newtonian scenario, for which Eddington luminosity of the radiation assures gravity-radiation balance at any radius. We explore properties of this relativistic equilibrium and the dynamics of test particles under radiation influence in the strong gravity regime.
\end{abstract}

Keywords. Test particle, radiation, general relativity, neutron stars

\section{Introduction}

A peculiar relativistic equilibrium of gravity and radiation was first indicated by Phinney (1987). Abramowicz et al. (1990) gave analytic expression for the radiation stressenergy tensor of static spherical central source, emitting radiation isotropically and homogeneously in its rest frame. Hence, their formulas apply to the case of Schwarzschild spacetime and so do the results presented in this paper. In recent years several groups pursued this subject in more details, investigating its aspects such as the drag forces dynamical importance, Bini et al. (2009), Oh et al. (2011), Stahl et al. (2012), possible astrophysical applications for bursts Stahl et al. (2013), Mishra \& Kluzniak (2014) and quasi-oscillatory behavior, Wielgus et al. (2012).

In this paper we recall the derivation of the equilibrium condition and investigate trajectories of test particles under radiation influence in curved Schwarzschild spacetime.

\section{Analytic formulation}

We begin with the Schwarzschild spacetime metric,

$$
\mathrm{d} s^{2}=-\xi \mathrm{d} t^{2}+\xi^{-1} \mathrm{~d} r^{2}+r^{2} \mathrm{~d} \theta^{2}+r^{2} \sin ^{2} \theta \mathrm{d} \phi^{2},
$$

and denote $\xi=1-2 / r$. We utilize geometric units $G=c=1$ and also put $M=1$, since the equations scale with mass of the central object. Assuming that the central mass (neutron star) is emitting radiation isotropically and homogeneously in its rest frame, the stress-energy tensor components in the static observer's orthonormal frame can be found by integrating specific intensity over the observer's local sky. The necessary calculations were performed by Abramowicz et al. (1990), yielding

$$
\begin{gathered}
T^{(t)(t)}=2 \pi I(r)\left(1-\cos \alpha_{0}\right), \\
T^{(t)(r)}=\pi I(r) \sin ^{2} \alpha_{0}
\end{gathered}
$$




$$
\begin{gathered}
T^{(r)(r)}=\frac{2}{3} \pi I(r)\left(1-\cos ^{3} \alpha_{0}\right), \\
T^{(\theta)(\theta)}=T^{(\phi)(\phi)}=\frac{1}{3} \pi I(r)\left(2-3 \cos \alpha_{0}+\cos ^{3} \alpha_{0}\right) .
\end{gathered}
$$

Static observer's orthonormal frame is denoted by indices in brackets. In Schwarzschild spacetime it is elementary to calculate coordinate frame components from orthonormal frame components by simple rescaling. $I(r)$ is a specific intensity, $R$ denotes radius of the star and $\alpha_{0}(r)$ is the star viewing angle,

$$
\begin{gathered}
I(r)=I(R)\left(\frac{1-2 / R}{\xi}\right)^{2}, \\
\alpha_{0}(r)=\arcsin \left[\frac{R}{r}\left(\frac{\xi}{1-2 / R}\right)^{1 / 2}\right] .
\end{gathered}
$$

Interestingly, similar analytic results were recently obtained assuming the observer located inside the luminous sphere in Schwarzschild spacetime. This case was derived by Wielgus \& Abramowicz (2015) and discussed in details by Wielgus et al. (2014), in this paper we focus on the more standard scenario of observer located outside of the luminous sphere. Knowing the radiation stress-energy tensor, we may evaluate the radiation flux, being

$$
F^{i}=-h_{j}^{i} T^{j k} u_{k},
$$

where $h^{i}{ }_{j}=\delta^{i}{ }_{j}+u^{i} u_{j}$ is the projection tensor and the corresponding four-force $f^{i}$ can be compared to the four-acceleration $a^{i}$, giving

$$
\frac{f^{i}}{m}=\frac{\sigma}{m} F^{i}=a^{i}=u^{k} \nabla_{k} u^{i}=\frac{\mathrm{d}^{2} x^{i}}{\mathrm{~d} s^{2}}+\Gamma_{j k}^{i} u^{j} u^{k} .
$$

Test particle effective cross section is denoted with $\sigma$ and its mass is denoted with $m$ (typically Thomson cross-section of electron and mass of a proton). Evaluating the Christoffel symbols, we find the equations of motion as functions for the proper time $\tau$

$$
\begin{gathered}
\frac{\mathrm{d}^{2} r}{\mathrm{~d} \tau^{2}}=(r-3)\left(\frac{\mathrm{d} \phi}{\mathrm{d} \tau}\right)^{2}-\frac{1}{r^{2}}+\frac{\sigma}{m} \xi T^{r t} u^{t}-\frac{\sigma}{m}\left(\xi^{-1} T^{r r}+T^{i j} u_{i} u_{j}\right) \frac{\mathrm{d} r}{\mathrm{~d} \tau}, \\
\frac{\mathrm{d}^{2} \phi}{\mathrm{d} \tau^{2}}=-\frac{2}{r} \frac{\mathrm{d} r}{\mathrm{~d} \tau} \frac{\mathrm{d} \phi}{\mathrm{d} \tau}-\frac{\sigma}{m}\left(r^{2} T^{\phi \phi}+T^{i k} u_{i} u_{k}\right) \frac{\mathrm{d} \phi}{\mathrm{d} \tau} .
\end{gathered}
$$

We identify four terms on the right hand side of Eq. 2.10 as, respectively: centrifugal acceleration, gravitational acceleration, radiation pressure, radial radiation drag. We also identify two terms on the right hand side of Eq. 2.11 as Coriolis acceleration and radiation azimuthal drag (Poynting-Robertson drag).

Putting all velocities to zero, we find the following critical point condition

$$
\frac{1}{r^{2}}=\frac{\sigma}{m} \xi^{1 / 2} T^{r t}
$$

Utilizing Eq. 2.3 and the classic Newtonian definition of the Eddington luminosity, $L_{\mathrm{Edd}}=4 \pi m / \sigma$, we find the critical point radial location

$$
r=R_{\mathrm{Edd}}=\frac{2}{1-\beta^{2}},
$$



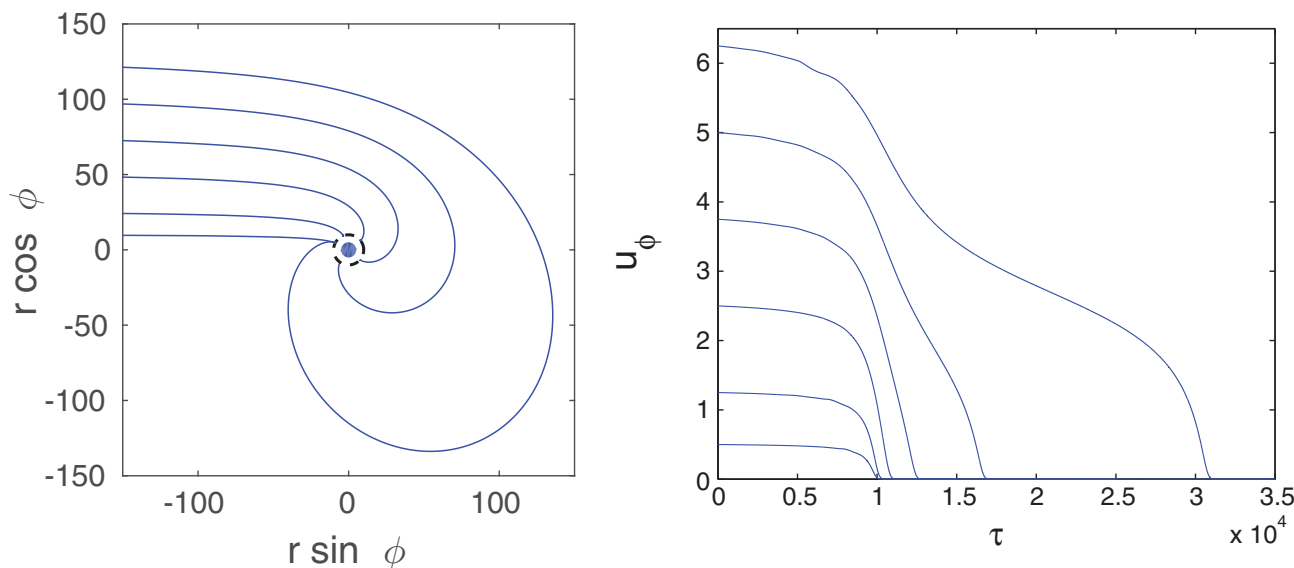

Figure 1. Left: trajectories of particles. Right: angular momentum $u_{\phi}$ variation along the trajectories.

where $\beta=L_{\infty} / L_{\mathrm{Edd}}$ and luminosity $L_{\infty}$ is

$$
L_{\infty}=\lim _{r \rightarrow \infty} 4 \pi r^{2} T^{t r} .
$$

Clearly, the ECS exists above the star surface for the range of $\beta$ parameter between $(1-2 / R)^{1 / 2}$ and $\beta=1$. The equilibrium of the Eddington Capture Sphere, at $r=R_{\mathrm{Edd}}$ is of a stable kind, net force always pointing towards the equilibrium. Radial and azimuthal drags work as efficient damping forces.

\section{Numerically calculated trajectories}

We give two numerical illustrations of the ECS related behavior of the test particles. To find the related trajectories, one needs to numerically integrate Eqs. 2.10-2.11. Following Stahl et al. (2012) we consider a stream of particles moving towards the luminous star (Hoyle-Lyttleton accretion). We observe that the typical behavior of the particles is to become captured by the ECS in a static equilibrium state, after drag forces remove most of the angular momentum. In the considered example, Fig. 1, we have $R_{\mathrm{Edd}}=15 \mathrm{M}$ and particles' velocity at infinity is equal to $0.05 \mathrm{c}, 6$ trajectories of different impact parameter $(10 M, 25 M, 50 M, 75 M, 100 M, 125 M)$ are shown. The right panel of Fig. 1 indicates, for the corresponding left panel trajectories, how effective is the angular momentum removing effect of drag forces in the strong radiation field. Because of the strong radiation and ECS presence, particles for significantly wider range of impact parameter can be captured. On the other hand, they remain levitating on the ECS rather than falling onto the star surface. This may result in cutting off the accretion-fueled radiation emission and hence destruction of the ECS, from which particles may infall and power up emission of radiation again. Such an oscillatory behavior was investigated by Wielgus et al. (2012).

The second numerical illustration concerns the stability of the static equilibrium on the ECS. We consider ECS located at $R_{\mathrm{Edd}}=10 M$ and test particles initially static on the ECS. Then we perturb the radial velocity, Fig. 2 left panel, or the azimuthal velocity, Fig. 2 right panel. The specific values of the perturbed velocities are $0.01 c, 0.05 c, 0.1 c, 0.2 c$ for the radial velocities and $0.01 c, 0.05 c, 0.1 c, 0.2 c, 0.3 c$ for the azimuthal velocities. Clearly, a very large initial velocity is necessary for the particle to escape the ECS and for all considered values the particle returned to the ECS. 

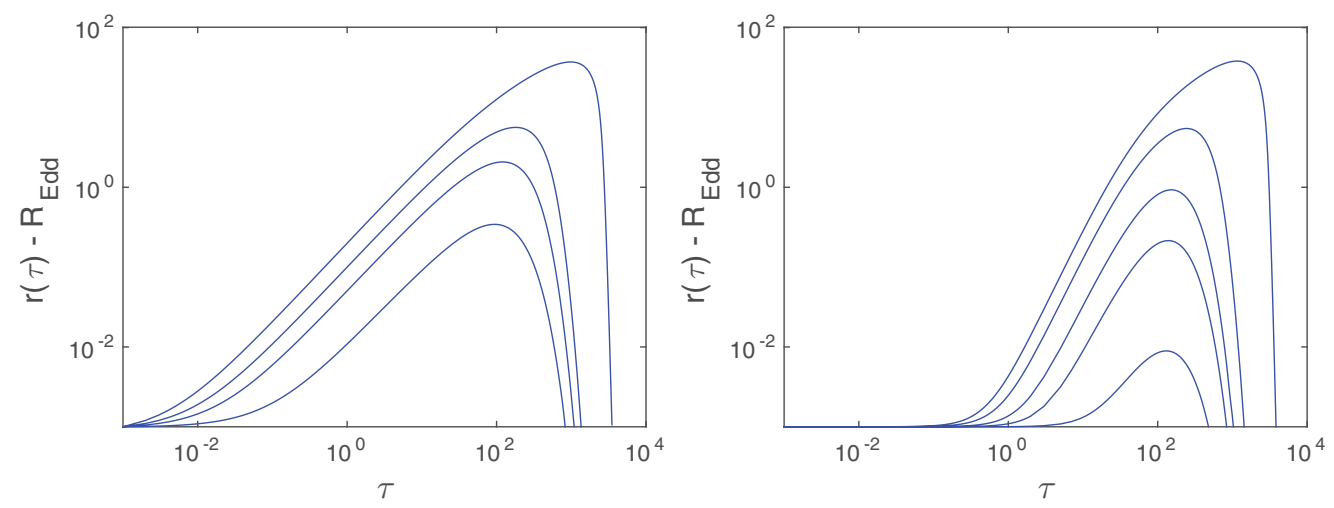

Figure 2. Left: particle radial location as function of proper time for perturbed radial velocity. Right: particle radial location as function of proper time for perturbed azimuthal velocity.

\section{Implications}

Spacetime around luminous relativistic star is divided into two regions, separated by the Eddington capture sphere. In the inner region radiation is effectively superEddingtonian, while outside of the ECS it is effectively sub-Eddingtonian. The radiationgravity balance is only achieved on the ECS. The ECS is an effect derived from the very first principles, and almost certainly (i.e., if general theory of relativity is correct) appears in Nature. More relevant question is whether there is some astrophysical relevance of this mechanism. It seems that at least in two contexts answer to this question should be positive. First is the quasioscillatory behavior related to the subsequent creation and disintegration of the ECS, Wielgus et al. (2012). The other is related to the dynamics of matter ejection from inner region of the superluminous system. Here the presence of the ECS and related radiation drag forces alter the ejected matter motion, e.g., Stahl et al. (2013).

\section{Acknowledgments}

The author thanks M. Abramowicz and W. Kluźniak for fruitful discussions. This research was supported by the Polish NCN grant UMO-2013/08/A/ST9/00795.

\section{References}

Abramowicz, M. A., Ellis, G. F. R., \& Lanza, A. 1990, ApJ, 361, 470

Bini, D., Jantzen, R. T., \& Stella, L. 2009, Class. Q. Grav., 26, 055009

Mishra, B. \& Kluzniak, W. 2013, A\&3A, 566, A62

Oh, J. S., Kim, H., \& Lee, H. M., 2011, New Astron., 16, 3, 183

Phinney, E. S. 1987, in Superluminal Radio Sources, eds. J. A. Zensus, \& T. J. Pearson (Cambridge: Cambridge University Press), 12

Stahl, A., Wielgus, M., Abramowicz, M. A., Kluzniak, W., \& Yu, W. 2012, A\&4A, 546, A54

Stahl, A., Kluzniak, W., Wielgus, M., \& Abramowicz, M. 2013, A\&A, 555, A114

Wielgus, M., Stahl, A., Abramowicz, M. A., \& Kluzniak, W. 2012, A\&̊A, 545, A123

Wielgus, M., Ellis, G. F. R., Vincent, F. H., \& Abramowicz, M. A. 2014, Phys. Rev. D, 90, 124024

Wielgus, M., \& Abramowicz, M., 2015, Proc. RAGtime, arXiv:1501.01540 